\title{
Response of Mycobacterium tuberculosis to Reactive Oxygen and Nitrogen Intermediates
}

\author{
Thomas R. Garbe, Nina S. Hibler, and V. Deretic \\ Department of Microbiology, University of Texas Health Science \\ Center at San Antonio, San Antonio, Texas, U.S.A.
}

\begin{abstract}
Background: Mycobacterium tuberculosis is a significant human pathogen capable of replicating in mononuclear phagocytic cells. Exposure to reactive oxygen and nitrogen intermediates is likely to represent an important aspect of the life cycle of this organism. The response of M. tuberculosis to these agents may be of significance for its survival in the host.

Materials and Methods: Patterns of de novo proteins synthesized in $M$. tuberculosis H37Rv exposed to compounds that generate reactive oxygen and nitrogen intermediates were studied by metabolic labeling and twodimensional electrophoresis.

Results: Menadione, a redox cycling compound which increases intracellular superoxide levels, caused enhanced synthesis of seven polypeptides, six of which appeared to be heat shock proteins. Chemical release of nitric oxide induced eight polypeptides of which only one could be identified as a heat shock protein. Nitric
\end{abstract}

oxide also exhibited a mild inhibitory action on general protein synthesis in the concentration range tested. Hydrogen peroxide did not cause differential gene expression and exerted a generalized inhibition in a dose-dependent manner. Cumene hydroperoxide caused mostly inhibition but induction of two heat shock proteins was detectable.

Conclusions: The presented findings indicate major differences between $M$. tuberculosis and the paradigms of oxidative stress response in enteric bacteria, and are consistent with the multiple lesions found in $\operatorname{oxy} R$ of this organism. The effect of hydrogen peroxide, which in Escherichia coli induces eight polypeptides known to be controlled by the central regulator $\operatorname{oxy} R$, appears to be absent in $M$. tuberculosis. Superoxide and nitric oxide responses, which in $E$. coli overlap and are controlled by the same regulatory system soxRS, represent discrete and independent phenomena in M. tuberculosis.

\section{INTRODUCTION}

Although tuberculosis has never ceased to remain a global problem (1), it has been considered a conquered disease in developed countries. However, a recent resurgence of tuberculosis in the United States and other industrialized nations, which is linked to acquired immunodeficiency syndrome (AIDS) and socio-economic factors (2), is a reminder that more needs to be learned about its etiologic agent, Mycobacterium tuberculosis. This pathogen is readily taken up by mononuclear phagocytes where it is capable of multiplying. Stimulation with cytokines enables macrophages to reduce their mycobacterial bur-

Address correspondence and reprint requests to: Thomas $\mathrm{R}$. Garbe, University of Texas Health Science Center at San Antonio, Department of Microbiology, 7703 Floyd Curl Drive, San Antonio, TX 78284-7739, U.S.A.

den (3-6), and based on experimental data it has been initially suggested that oxygen radicals are responsible for the intracellular killing of $M$. $t u$ berculosis (7-9). More recent investigations however have shifted the emphasis from reactive oxygen species to reactive nitrogen intermediates (3-6), but perhaps both types of radicals are involved, as exemplified by hybrid products such as peroxynitrite (10).

In enteric bacteria, which currently serve as model systems for oxidative stress response $(11,12)$, exposure to superoxide $\left(\mathrm{O}_{2}{ }^{-} \cdot\right)$ generating compounds and hydrogen peroxide induces or enhances expression of over 90 genes and is controlled by several central regulators, two of which, OxyR and SoxRS, have been identified and thoroughly characterized (13-16) (Fig. 1A). The oxyR regulon is activated upon exposure to 

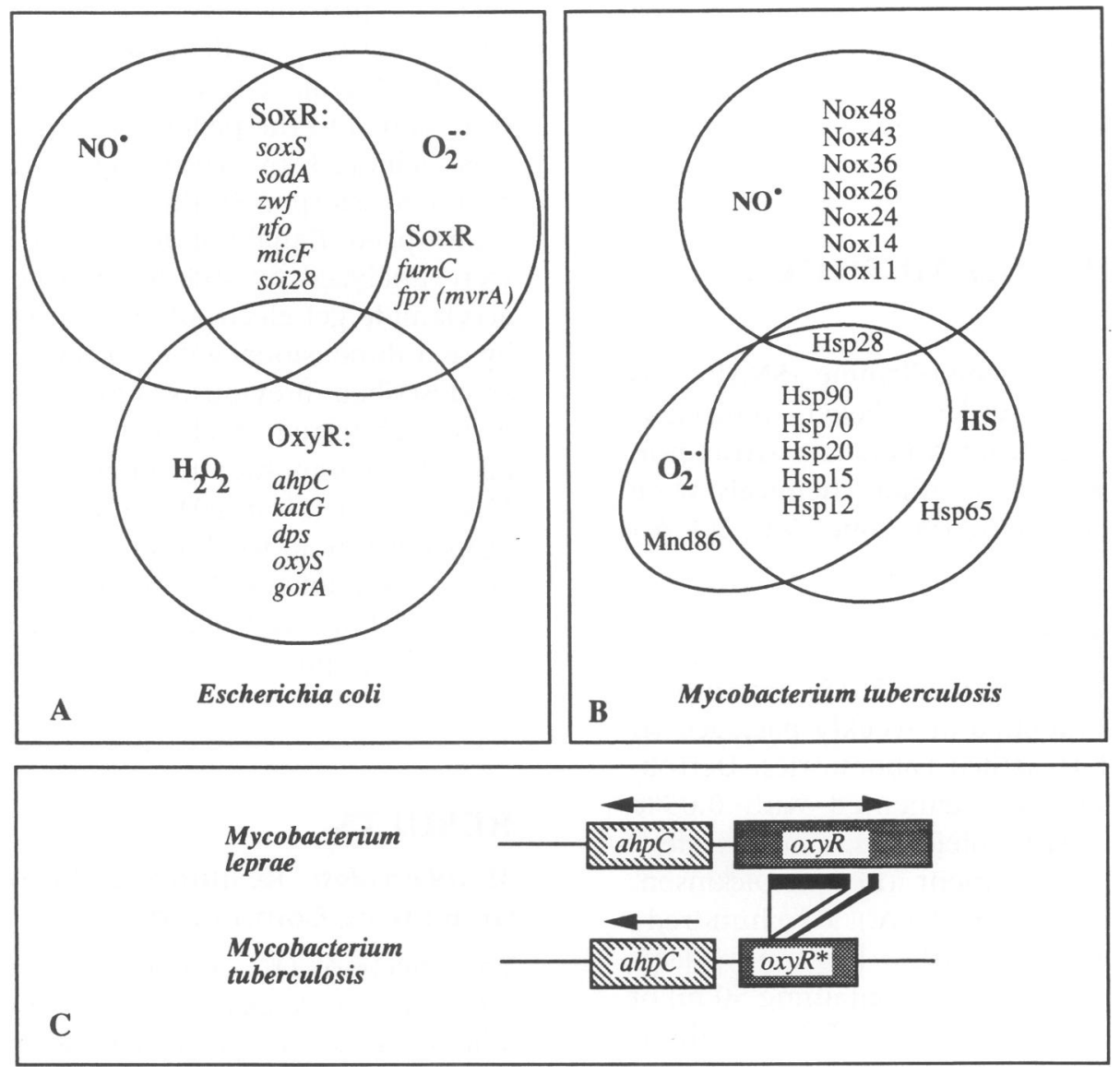

FIG. 1. Contrasting patterns of stress response in enteric bacteria and $M$. tuberculosis

The idiosyncrasies of stress response (circles) in enteric bacteria (A) and M. tuberculosis (B) can be summarized as follows: (i) No significant overlap between $\mathrm{O}_{2}^{-}$. and NO regulon in M. tuberculosis. (ii) The majority of $\mathrm{O}_{2}{ }^{-\cdot}$ induced polypeptides in $M$. tuberculosis are the previously characterized heat shock proteins (Hsp), with the exception of the newly identified $\mathrm{O}_{2}{ }^{-\cdot}$ inducible polypeptide $\mathrm{Mnd86}$. (iii) $\mathrm{NO}^{\cdot}$ induces a number of polypeptides (designated as Nox followed by apparent $\mathrm{M}_{\mathrm{r}}$ ) that appear not to be inducible by other stress factors tested. (iv) $M$. tuberculosis has multiple lesions in $\operatorname{oxy} R(24)$ as illustrated in Panel C (comparison with a functional oxyR gene from $M$. leprae; deletions are marked as filled rectangles). Consistent with these findings, there is no differential induction of gene expression in $M$. tuberculosis exposed to $\mathrm{H}_{2} \mathrm{O}_{2}$ except in the case of kat $G$, which appears to be affected in combination with other cultural conditions (e.g., slightly elevated temperature) (24). HS, heat shock. Genes and protein names are explained in the text.

hydrogen peroxide and leads to increased synthesis of nine proteins, including catalase-peroxidase (KatG), glutathione reductase (GorA), alkyl hydroperoxide reductase (Ahp), and Dps, a nonspecific DNA-binding protein (17). The soxRS regulon in Escherichia coli is activated by superoxide generating compounds (15), nitric oxide (NO) $(18,19)$, and by several oxidants even under anaerobic conditions $(20,21)$. It has been suggested that the central regulator SoxR, which is an iron-sulfur protein (22), responds to the balance between reduced and oxidized flavodoxin and ferredoxin (21). This balance is disturbed by redox cycling compounds such as menadione, which divert electrons from NADPH to the reduction of oxygen to generate $\mathrm{O}_{2}{ }^{-\cdot} \cdot(21)$, resulting in the activation of at least 10 genes (15). These include the genes encoding Mn-containing superoxide dismutase $(\operatorname{sod} A)$, glucose 6-phosphate dehydrogenase (zwf), NADPH: ferredoxin oxidoreductase $(f p r)(21)$, DNA repair endonuclease IV $(n f o)$, and stable fumarase ( fumC) (23).

We show here that the response of $M$. tuberculosis to oxidants differs from the response seen in E. coli and Salmonella typhymurium (Fig. 1B). These findings are consistent with the recent data indicating that the $M$. tuberculosis analog of 
oxy $R$ is inactivated by multiple mutations (24) (Fig. 1C), and suggest further differences between this organism and the currently known models of oxidative stress in enteric bacteria.

\section{MATERIALS AND METHODS}

\section{Chemicals}

S-nitroso-N-acetyl-D,L-penicillamine (SNAP) was from Alexis. Methyl- ${ }^{14} \mathrm{C}$-labeled molecular weight marker was from Amersham (Arlington Heights, IL, U.S.A.). All other chemicals were from Sigma Chemical Co. (St. Louis, MO, U.S.A.)

\section{Cultures and Strains}

M. tuberculosis H37Rv (ATCC 25618), was grown at $37^{\circ} \mathrm{C}$ and maintained at weekly passages on Middlebrook 7H10 (Difco Laboratories, Detroit, MI, U.S.A.) plates supplemented with $0.05 \%$ Tween 80 and OADC (oleic acid, albumin, dextrose, catalase) enrichment (Becton-Dickinson, BBL, Mountain View, CA, U.S.A.). Two hundredmilliliter polystyrene bottles (Corning Glassworks, Corning, NY, U.S.A.) containing $50 \mathrm{ml}$ of Middlebrook 7H9 basal medium (Difco) without glycerol and detergent, supplemented with either ADC (albumin, dextrose, catalase) enrichment (Difco) or ADc (albumin-dextrose complex) enrichment, were inoculated with a loopful of bacterial material and incubated at $37^{\circ} \mathrm{C}$ on a continuous roller. ADc contained 5\% bovine albumin fraction V (supplied by Boehringer Mannheim, Indianapolis, IN, U.S.A.), $2 \%$ glucose, and $0.85 \%$ sodium chloride and was filtrated through a $0.22-\mu \mathrm{m}$ membrane.

\section{Metabolic Labeling and Two-Dimensional Gel Electrophoresis}

M. tuberculosis cultures were incubated by continuous rolling for 3 days or as indicated and then used for labeling after pelleting by centrifugation at $3,000 \times g$ for $5 \mathrm{~min}$ and $20^{\circ} \mathrm{C}$ and resuspending in $1 / 7$ volume of the original growth medium. One-milliliter aliquots were exposed to tested compounds by adding 1 to $10 \mu$ l of solutions in water (hydrogen peroxide) or dimethyl sulfoxide (all other compounds) followed by addition of 10 $\mu \mathrm{Ci}{ }^{35} \mathrm{~S}$-methionine and ${ }^{35} \mathrm{~S}$-cysteine (NEN, protein labeling mix). After $4 \mathrm{hr}$ of rolling incubation at $37^{\circ} \mathrm{C}$, the samples were spun for $3 \mathrm{~min}$ in an Eppendorf microcentrifuge at maximum speed. Approximately $50 \mu \mathrm{l}$ of zirconium beads (0.1- to $0.15-\mathrm{mm}$ diameter, Biospec Products, Inc., Bartlesville, OK, U.S.A.) and $100 \mu \mathrm{l}$ of water were added to the pellets. Disruption of bacteria was achieved by vortexing in a mini-beadbeater- 8 (Biospec Products) for 2 min at maximum speed. Equal volumes of the homogenates were analyzed by sodium dodecylsulfate-polyacrylamide gel electrophoresis (SDS-PAGE) and by two-dimensional gel (2-D gel) electrophoresis as described previously $(25,26)$. The proteins were transferred by electroblotting onto Immobilon-P membrane (Millipore, Marlborough, MA, U.S.A.) in $10 \mathrm{mM}$ 3-[cyclohexylamino]-1propanesulfonic acid buffer at $\mathrm{pH} 11(\mathrm{NaOH})$ and $10 \%$ methanol at either 5 volts over night or 50 volts for $\mathrm{l} \mathrm{hr}$, and the filters were subjected to autoradiography.

\section{RESULTS}

\section{M. tuberculosis Response to Superoxide Generating Compounds}

The effects of $\mathrm{O}_{2}^{-}$. on protein synthesis in $M$. tuberculosis were examined by exposure to menadione, a redox cycling compound which increases intracellular levels of $\mathrm{O}_{2}^{-\cdot}(21,27,28)$. Samples from cultures treated with menadione concentrations that caused differential display of newly synthesized polypeptides by SDS-PAGE analysis were further examined by electrophoresis in 2-D gels. Several phenomena were observed: (i) The response to menadione was dependent on the growth stage. The two cultures shown in Figs. 2 and 3 were in different growth stages as noticeable by the differences in the patterns of de novo synthesized polypeptides seen in Panels A (Figs. 2 and 3), representing the two untreated control cultures. (ii) In the menadione treated samples, qualitative differences in the polypeptide induction and overall inhibition of protein synthesis were seen in the 3- and 7-day cultures. (iii) Most of the inducible proteins overlapped with several characterized heat shock proteins (26), namely Hsp15, Hsp28, Hsp70 (DnaK), and Hsp90 (Figs. 2 and 3). The up-regulated 20-kD polypeptide in Figs. $2 \mathrm{~B}$ and $3 \mathrm{~B}$ is most likely the previously identified Hsp20 (26). (iv) Occasionally, exposure to $300 \mu \mathrm{M}$ menadione induced an $86-\mathrm{kD}$ polypeptide (Mnd86) (data not shown). Since this polypeptide overlapped with the position in 2-D gels of a polypeptide reactive with polyclonal antibodies 

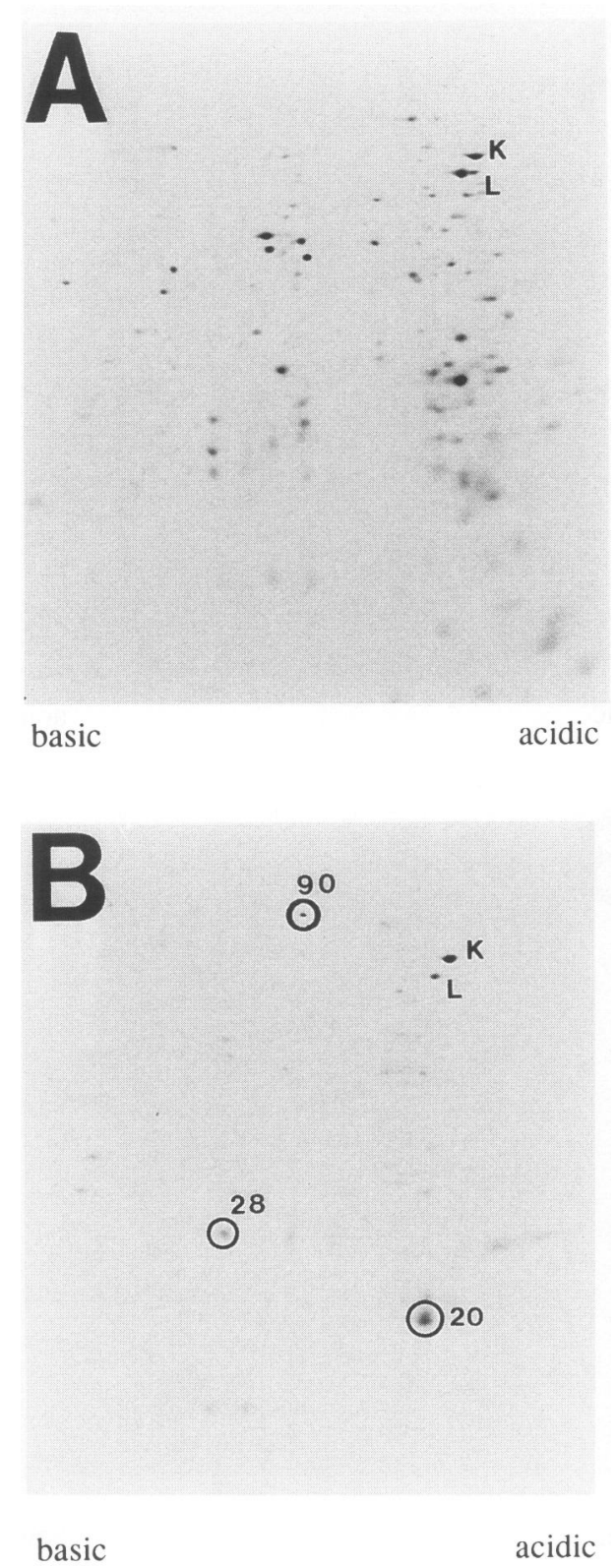

FIG. 2. Early growth phase response in $M$. $t u$ berculosis to menadione

Autoradiograms show 2-D gel profiles of metabolically labelled proteins in aliquots of a 3-day M. tuberculosis H37Rv culture in 7H9+ADc medium without any addition (A) and with $300 \mu \mathrm{M}$ menadione, a superoxide generating redox cycling compound (B). Circles indicate induced polypeptides and numbers indicate their apparent molecular weights. $\mathrm{K}$, DnaK (Hsp70) (26); L, GroEL (Hsp65) (26,35). DnaK is also up-regulated by menadione.
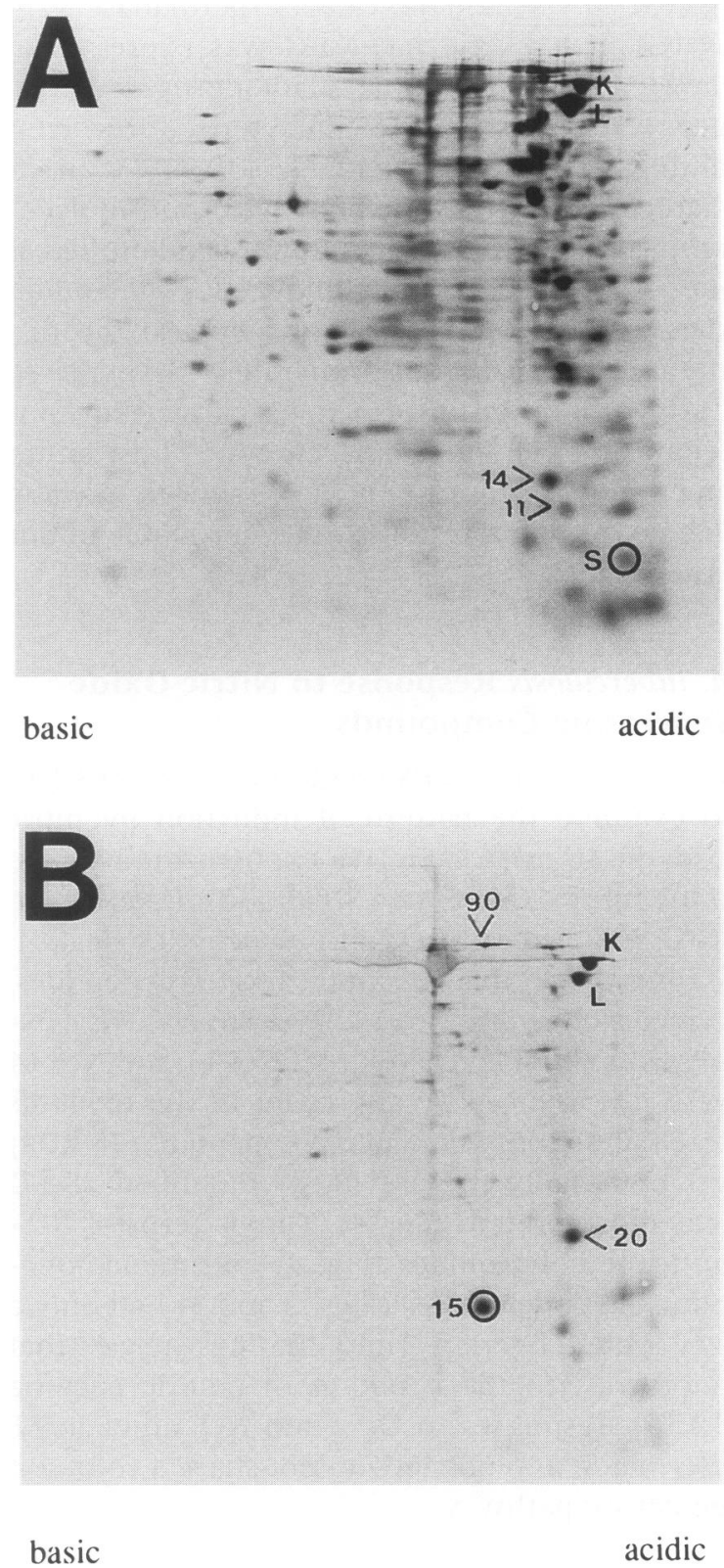

FIG. 3. Late growth-phase response of $M$. tuberculosis to menadione

Autoradiographs from 2-D gels show profiles of metabolically labeled polypeptides of aliquots from a 6-day $M$. tuberculosis $\mathrm{H} 37 \mathrm{Rv}$ culture in 7H9+ADc medium (A) and with $300 \mu \mathrm{M}$ menadione (B). Several prominent polypeptides are highlighted and numbers indicate their apparent molecular weights. Polypeptides 14 and $11 \mathrm{kD}$ in Panel A are identical with Noxl4 and Nox11 of Fig. 4 and are repressed by menadione (B). The circled spot $S$ overlaps with one of the major spots that occasionally show up by total protein stain (data not shown) and is possibly Hsp GroES, as judged by position and earlier published data (26). Other markings as in Fig. 2. 
against $M$. tuberculosis catalase-peroxidase (29) (KatG), it is likely that KatG was expressed at elevated levels in some menadione-treated samples. (v) The previously characterized iron-containing superoxide dismutase $(30,31)$ with its characteristic isoelectric point (26) and molecular mass of $23 \mathrm{kD}(30)$, could not be identified as one of the differentially induced polypeptides (Figs. 2 and 3). Furthermore, superoxide dismutase activity did not comigrate with increased ${ }^{35} \mathrm{~S}$ incorporation in proteins separated in nondenaturing gels (data not shown). Thus, either superoxide dismutase is not induced or any change may be too subtle to be detectable in our experiments.

\section{M. tuberculosis Response to Nitric Oxide Generating Compounds}

NO' activates the soxRS regulon in E. coli $(18,19)$. To examine the pattern of induction by nitric oxide in $M$. tuberculosis, we exposed the bacteria to the nitric oxide donor SNAP (32). Exposure to SNAP induced a variety of polypeptides (Fig. 4). Among these, the 11- and 14-kD polypeptides, termed Nox 11 and Nox 14, respectively, were expressed at high levels (Fig. 4). Noxl1 and Noxl4 were not induced by any other of the oxidants tested. Only one inducible polypeptide $(28 \mathrm{kDa})$ was potentially the previously identified $28 \mathrm{-kD}$ heat shock protein (Fig. 4). The patterns of NOinducible polypeptides (Fig. 4) and menadioneinducible polypeptides (Figs. 2 and 3 ) had only a very limited overlap. These findings suggest that in contrast to the situation in enteric bacteria $(18,19)$, responses to $\mathrm{O}_{2}{ }^{-}$and $\mathrm{NO}^{-}$differ in $M$. tuberculosis, and possibly do not share a common regulatory pathway.

\section{M. tuberculosis Response to Hydrogen Peroxide and Organic Peroxides}

Exposure of M. tuberculosis to a range of hydrogen peroxide concentrations did not display any differential gene expression as judged by SDSPAGE (Fig. 5) and by patterns of de novo protein synthesis in 2-D gels (not shown). In contrast to E. coli and S. typhimurium, where hydrogen peroxide enhances synthesis of at least 30 polypeptides (16), the effect of hydrogen peroxide on $M$. tuberculosis was that of a generalized inhibition, and synthesis of most polypeptides ceased in a dose-dependent manner. Inhibition was clearly visible at concentrations as low as $100 \mu \mathrm{M}$ (Fig. 5). Analysis by 2-D gel electrophoresis
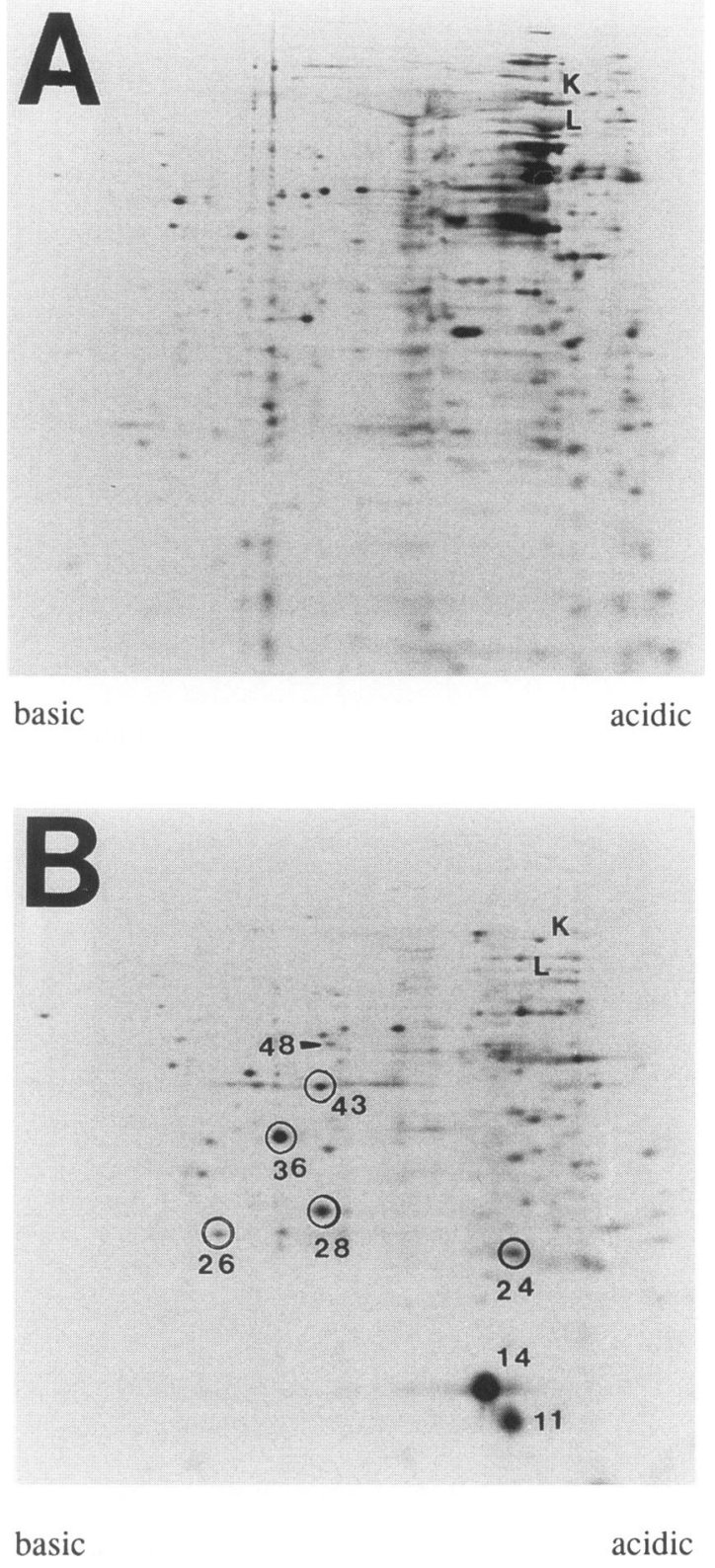

FIG. 4. M. tuberculosis response to nitric oxide generating compound SNAP

Autoradiograms of 2-D gels show newly synthesized proteins in aliquots of a 3-day M. tuberculosis $\mathrm{H} 37 \mathrm{Rv}$ culture in 7H9+ADc medium. (A) Control (no additions). (B) $100 \mu \mathrm{M}$ SNAP, an NO'-generating compound. Circles or wedges highlight the most prominently induced polypeptides and numbers indicate their apparent molecular weights. Reference to these polypeptides is given in the text by the prefix Nox. $\mathrm{K}$ and $\mathrm{L}$, as in Figs. 2 and 3.

showed no differential induction of polypeptides upon exposure to hydrogen peroxide with the exception of a variable presence of a polypeptide with an apparent $M_{r}$ similar to the observed $M_{r}$ of Mnd86 (data not shown). In our previous 


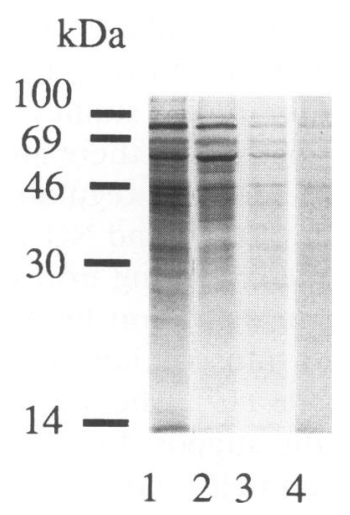

FIG. 5. Effects of hydrogen peroxide on protein synthesis in $M$. tuberculosis

SDS-PAGE autoradiogram shows metabolically labeled polypeptides in aliquots taken from a three day culture of $M$. tuberculosis $\mathrm{H} 37 \mathrm{Rv}$ in 7H9+ADc exposed to increasing amounts of hydrogen peroxide. Lanes: 1 , control; 2-4, $0.1 \mu \mathrm{M} ; 1 \mu \mathrm{M}$; and 10 $\mu \mathrm{M}$ hydrogen peroxide. Exposure of Lane 4 was twice as long as the other lanes.

report on katG studies (24), we could detect a 2 -fold induction of the corresponding polypeptide only under slightly elevated growth temperatures. The overall lack of response to hydrogen peroxide is in sharp contrast to the reaction of enteric bacteria. These findings appear to be con- sistent with the recent observation that the putative oxyR gene of $M$. tuberculosis has an incomplete open reading frame and is most likely inactive (24).

To probe the response of the tubercle bacillus to organic peroxides, we employed cumene hydroperoxide. In repeated experiments, this compound proved to be more inhibitory than hydrogen peroxide. However, in contrast to hydrogen peroxide, a concentration of cumene hydroperoxide was found showing a pattern consistent with differential gene expression. The results of a titration experiment with cumene hydroperoxide, analyzed by SDS-PAGE, are shown in Fig. 6A. Induction of a $15-$ and a $28-\mathrm{kD}$ polypeptide occurred at $200 \mu \mathrm{M}$ cumene hydroperoxide and was further enhanced at $500 \mu \mathrm{M}$. This effect was not visible at $100 \mu \mathrm{M}$, a concentration that was nevertheless inhibitory for protein synthesis. Further analysis of the inducible polypeptides by 2-D gel electrophoresis yielded a distinct pattern depicted in Fig. 6B. The cumene hydroperoxideinducible 15 - and 28-kD polypeptides overlap with the positions of the previously characterized heat shock proteins Hspl5 and Hsp28 (26). Figure 6B also shows 45- and 53-kD polypeptides (Chp45 and Chp53), not discernible on single dimension SDS-PAGE autoradiograms (Fig. 6A).

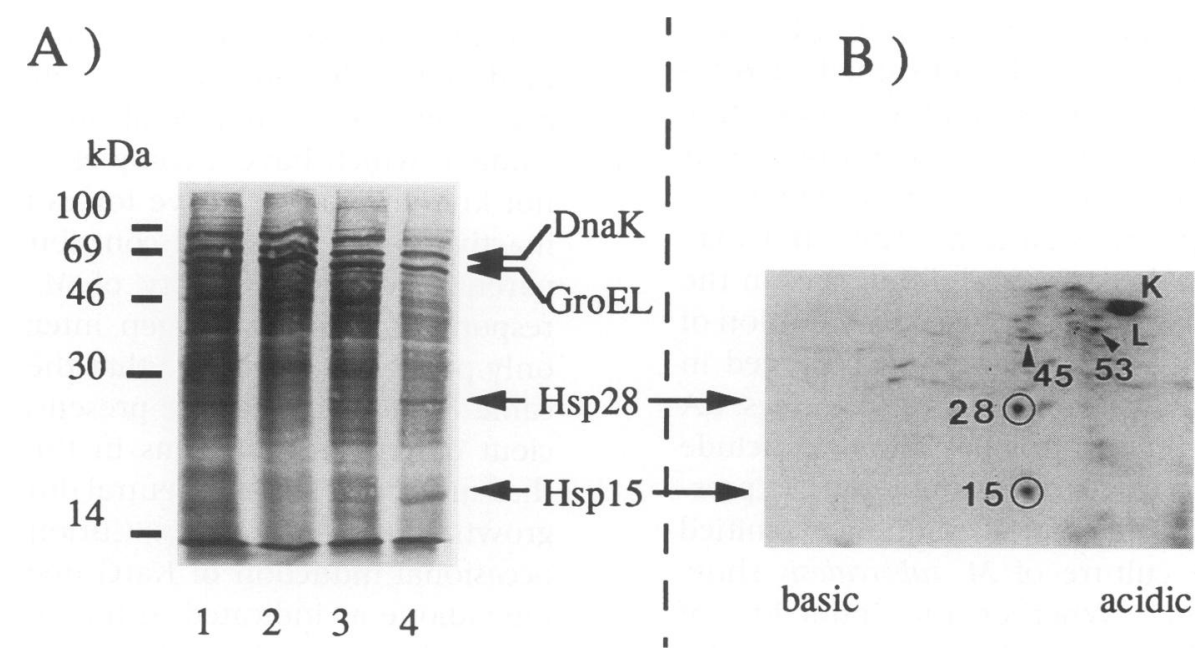

FIG. 6. M. tuberculosis response to cumene hydroperoxide

(A) SDS-PAGE analysis of metabolically labelled polypeptides in aliquots from culture grown in $7 \mathrm{H} 9+\mathrm{ADC}$ medium exposed to increasing amounts of cumene hydroperoxide. Lanes: $1,37^{\circ} \mathrm{C}$ control; $2-4,100 \mu \mathrm{M} ; 200 \mu \mathrm{M}$; and $500 \mu \mathrm{M}$ cumene hydroperoxide. (B) Analysis by 2-D gel electrophoresis of the $500 \mu \mathrm{M}$ cumene hydroperoxide treated sample (Panel A, Lane 4). Two major induced polypeptides are labeled as heat shock proteins 15 and 28 on the basis of their positions and earlier published data (26). The 45- and 53-kD polypeptides are referred to in the text as Chp45 and Chp53. DnaK and GroEL (Hsp70 and Hsp65) are indicated as markers to facilitate comparisons. 


\section{DISCUSSION}

In this work, the patterns of gene expression in $M$. tuberculosis in response to challenges with reactive oxygen and nitrogen intermediates have been examined and compared. Several conclusions can be drawn: (i) M. tuberculosis has a very limited response to hydrogen peroxide which is in agreement with the recently reported naturally occurring lesions in the putative oxyR gene of M. tuberculosis (24) (Fig. 1C). (ii) Exposure to redox cycling agents, which increase endogenously generated $\mathrm{O}_{2}{ }^{-}$, causes induction of a number of gene products. However, the majority of these polypeptides appear to overlap with the heat shock proteins (Fig. 1B). (iii) A vigorous and complex display of differential gene expression is observed upon exposure to $\mathrm{NO}^{\circ}$ generating compounds. (iv) There is very little overlap between $\mathrm{O}_{2}{ }^{-} \cdot$-induced response and heat shock proteins on one side and $\mathrm{NO}^{-}$-induced polypeptides on the other (Fig. 1B), suggesting that they may be controlled by independent regulatory mechanisms.

Perhaps the most intriguing observation presented here is the differential display of gene expression in response to nitric oxide. Preliminary studies with $\mathrm{NO}^{\circ}$-generating compounds other than SNAP (e.g., nitroso glutathione, diethylenetriamine nitric oxide adduct) support the data shown in this work. $\mathrm{NO}^{\circ}$ gas was not used in the present study, since a recent report (19) indicates that a slow but continued release of $\mathrm{NO}^{\circ}$ has a more profound effect than a bolus of $\mathrm{NO}^{\circ}$, which may be quickly consumed in reactions with oxygen. Using several $\mathrm{NO}^{\circ}$ generating reagents we have observed induction of similar polypeptides but also noticed some variability in 2-D gel electrophoresis patterns. The latter may be partly due to the structural differences in the tested chemicals but is also caused by variation of metabolic activity in $M$. tuberculosis observed in parallel untreated control cultures (e.g., Figs. 2A and 4A). Nevertheless, it is possible to conclude that nitric oxide elicits differential gene expression in this organism since all aliquots examined from the same culture of $M$. tuberculosis show consistent results. Whether the induction of Nox11, Nox 14, and additional polypeptides (Fig. 1B) is related to the pathogenesis and virulence of $M$. tuberculosis is currently under investigation. The most prominent polypeptides induced by all NO generating compounds tested are being further characterized.

Another observation of potential relevance for the idiosyncrasies of oxidative stress response in $M$. tuberculosis is the difference in patterns of $\mathrm{O}_{2}{ }^{-}$- and $\mathrm{NO}^{-}$-induced polypeptides. In contrast to this observation, recent studies in $E$. coli indicate that in this bacterium there are major overlaps between $\mathrm{O}_{2}^{-} \cdot$ and $\mathrm{NO}^{-}$regulons (Fig. 1). The central regulator of $\mathrm{O}_{2}{ }^{-}$and $\mathrm{NO}^{-}$response in $E$. coli is SoxR, a redox sensing iron-sulfur protein (22) which, upon activation by a redox signal, causes increased transcription of 10 genes via a regulatory cascade (19). The analyses presented here provide little support for possible overlaps between $\mathrm{O}_{2}{ }^{-}$- and $\mathrm{NO}^{-}$-inducible systems in $M$. tuberculosis. It is also worth noting the conspicuous absence of detectable induction of superoxide dismutase upon exposure to menadione. Furthermore, six of the seven polypeptides consistently inducible with menadione appear to be heat shock proteins. One possible explanation for this phenomenon is that the effects of $\mathrm{O}_{2}{ }^{-}$on gene expression may be indirect, i.e., via protein denaturation (33), a process suggested to induce heat shock response (34).

The observations reported here and elsewhere (24) suggest that $M$. tuberculosis and other members of the $M$. tuberculosis complex have undergone a partial loss of response to oxidative stress in the course of their evolution. Some vestiges of the tubercle bacillus ancestor's capacity to adjust to the stress imposed by reactive oxygen intermediates can be seen by the presence of an inactive $\operatorname{oxy} R$ gene upstream of $\operatorname{ahpC}(24)$. The lesions in $\operatorname{oxy} R$ have been observed in all strains and members of $M$. tuberculosis complex examined to date, in contrast to the situation in $\mathrm{Myco}$ bacterium leprae and several other mycobacteria studied, which have a complete $\operatorname{oxy} R$ gene. It is not known what selective forces resulted in the inactivation of $\operatorname{xy} R$ and contributed to the apparently reduced capacity of $M$. tuberculosis to respond to reactive oxygen intermediates. It is only possible to speculate that these systems became obsolete due to the presence of more efficient defense mechanisms in this organism, or that such processes are neutral during the in vivo growth of $M$. tuberculosis. Curiously, we found occasional induction of KatG upon exposure to menadione as indicated in this work and also a slight change with hydrogen peroxide, most prominently observed under growth at elevated temperatures (24). However, the detected increases in newly synthesized KatG appear to be sensitive to small variations in culture conditions, and any conclusion regarding potential regulation must await further genetic investigations. 
In contrast to the apparent lack of specific response to reactive oxygen intermediates is the synthesis of at least seven novel proteins in response to NO. While the role of this radical remains controversial for the infections in humans, members of the $M$. tuberculosis complex infect other host species that have a demonstrable inducible NO synthase in macrophages. Furthermore, several studies (3-6) suggest that $\mathrm{NO}^{\circ}$ mediated killing of intracellular $M$. tuberculosis takes place in macrophages from such sources. Whether the members of the M. tuberculosis complex have evolved specific regulatory and defense mechanisms to cope with exposure to $\mathrm{NO}^{\circ}$ is currently under investigation.

\section{ACKNOWLEDGMENTS}

Funding for this work was provided by National Institutes of Health Public Health Service Grant AI35217.

\section{REFERENCES}

1. Kochi A. (1991) The global tuberculosis situation and the new control strategy of the World Health Organization. Tubercle 72: 1-6.

2. Murray JF, Mills J. (1990) Pulmonary infectious complications of human immunodeficiency virus infection. Am. Rev. Respir. Dis. 141: 1356-1372.

3. Flesch I, Kaufmann SHE. (1991) Mechanisms involved in mycobacterial growth inhibition by gamma interferon-activated bone marrow macrophages: Role of reactive nitrogen intermediates. Infect. Immun. 59: 3213-3218.

4. Denis M. (1991) Interferon-gamma-treated murine macrophages inhibit growth of tubercle bacilli via the generation of reactive nitrogen intermediates. Cell. Immunol. 132: 150-157.

5. Chan J, Xing J, Magliozzo RS, Bloom BR. (1992) Killing of virulent Mycobacterium tuberculosis by reactive nitrogen intermediates produced by activated murine macrophages. J. Exp. Med. 175: 1111-1122.

6. Chan J, Tanaka K, Carroll D, Flynn J, Bloom BR. (1995) Effects of nitric oxide synthase inhibitors on murine infection with Mycobacterium tuberculosis. Infect. Immun. 63: 736740.

7. Jackett PS, Aber VR, Mitchison DA, Lowry
DB. (1981) The contribution of hydrogen peroxide resistance to virulence of Mycobacterium tuberculosis during the first six days after intravenous infection of normal and BCG-vaccinated guinea pigs. $B r . J$. Exp. Pathol. 62: 34-40.

8. Jackett PS, Andrew PW, Aber VR, Lowrie DB. (1983) Guinea pig alveolar macrophages probably kill $M$. tuberculosis H37Rv and H37Ra in vivo by producing hydrogen peroxide. Adv. Exp. Med. Biol. 162: 99-104.

9. Walker L, Lowrie DB. (1981) Killing of $M y$ cobacterium microti by immunologically activated macrophages. Nature (Lond.) 293: 6970.

10. Zhu L, Gunn C, Beckman JS. (1992) Bactericidal activity of peroxynitrite. Arch. Biochem. Biophys. 298: 452-457.

11. Farr SB, Kogoma T. (1991) Oxidative stress responses in Escherichia coli and Salmonella typhimurium. Microbiol. Rev. 55: 561-585.

12. Demple B, Amábile-Cuevas CF. (1991) Redox redux: The control of oxidative stress responses. Cell 67: 837-839.

13. Christman MF, Morgan RW, Jacobson FS, Ames BN. (1985) Positive control of a regulon for defenses against oxidative stress and some heat-shock proteins in Salmonella typhimurium. Cell 41: 753-762.

14. Tsaneva IR, Weiss B. (1990) soxR, a locus governing a superoxide response regulon in Escherichia coli K-12. J. Bacteriol. 172: 41974205.

15. Amábile-Cuevas CF, Demple B. (1991) Molecular characterization of the soxRS genes of Escherichia coli: two genes control a superoxide stress regulon. Nucleic Acids Res. 19: 4479-4484.

16. Storz G, Altuvia S. (1994) OxyR regulon. Methods Enzymol. 234: 217-223.

17. Altuvia S, Almiron M, Huisman G, Kolter R, Storz G. (1994) The dps promoter is activated by OxyR during growth and by IHF and $s^{s}$ in stationary phase. Mol. Microbiol. 13: 265-272.

18. Nunoshiba T, DeRojas-Walker T, Wishnok JS, Tannenbaum SR, Demple B. (1993) Activation by nitric oxide of an oxidative-stress response that defends Escherichia coli against activated macrophages. Proc. Natl. Acad. Sci. U.S.A. 90: 9993-9997.

19. Nunoshiba T, DeRojas-Walker T, Tannenbaum RS, Demple B. (1995) Roles of nitric oxide in inducible resistance of Escherichia coli to activated murine macrophages. Infect. Immun. 63: 794-798. 
20. Privalle CT, Fridovich I. (1990) Anaerobic biosynthesis of the manganese-containing superoxide dismutase in Escherichia coli. Effects of diazenedicarboxylic acid bis $\left(\mathrm{N}, \mathrm{N}^{\prime}-\right.$ dimethylamide) (diamide). J. Biol. Chem. 265: 21966-21970.

21. Liochev SI, Hausladen A, Beyer Jr WF, Fridovich I. (1994) NADPH:ferredoxin oxidoreductase acts as a paraquat diaphorase and is a member of the soxRS regulon. Proc. Natl. Acad. Sci. U.S.A. 91: 1328-1331.

22. Hidalgo E, Demple B. (1994) An iron-sulfur center essential for transcriptional activation by the redox-sensing SoxR protein. EMBO J. 13: $138-146$.

23. Liochev SI, Fridovich I. (1992) Fumarase C, the stable fumarase of Escherichia coli, is controlled by the soxRS regulon. Proc. Natl. Acad. Sci. U.S.A. 89: 5892-5896.

24. Deretic V, Philipp W, Dhandayuthapani S, et al. (1995) Mycobacterium tuberculosis is a natural mutant with inactivated oxidative stress regulatory gene: Implications for sensitivity to isoniazid. Mol. Microbiol. 17: 889-900.

25. O'Farrell PZ, Goodman HM, O'Farrell PH. (1977) High resolution two-dimensional electrophoresis of basic as well as acidic proteins. Cell 12: 1133-1142.

26. Young DB, Garbe TR. (1991) Heat shock proteins and antigens of Mycobacterium tuberculosis. Infect. Immun. 59: 3086-3093.

27. Walkup L, Kogoma T. (1989) Escherichia coli proteins inducible by oxidative stress mediated by superoxide radical. J. Bacteriol. 171: 1476-1484.

28. Greenberg JT, Demple B. (1989) A global response induced in Escherichia coli by redox-

Contributed by S. Falkow on October 2, 1995. cycling agents overlaps with that induced by peroxide stress. J. Bacteriol. 171: 39333939.

29. Heym B, Zhang Y, Poulet S, Young D, Cole ST. (1993) Characterization of the kat Gene encoding a catalase-peroxidase required for isoniazid susceptibility of Mycobacterium tuberculosis. J. Bacteriol. 175: 4255-4259.

30. Zhang Y, Lathigra R, Garbe T, Catty D, Young D. (1991) Genetic analysis of superoxide dismutase, the 23 kilodalton antigen of Mycobacterium tuberculosis. Mol. Microbiol. 5: 381-391.

31. Cooper JB, McIntyre K, Badasso MO, et al. (1995) X-ray structure analysis of the irondependent superoxide dismutase from $\mathrm{Myco}$ bacterium tuberculosis at 2.0 Ångstroms resolution reveals novel dimer-dimer interactions. J. Mol. Biol. 246: 531-544.

32. Gibson A, Babbedge R, Brave SR, et al. (1992) An investigation of some S-nitrosothiols, and of hydroxy-arginine, on the mouse anococcygeus. Br. J. Pharmacol. 107: 715-721.

33. Weiner L, Kreimer D, Roth E, Silman I. (1994) Oxidative stress transforms acetylcholinesterase to a molten-globule-like state. Biochem. Biophys. Res. Commun. 198: 915-922.

34. Bukau B. (1993) Regulation of the Escherichia coli heat shock response. Mol. Microbiol. 9: 671-680.

35. Kong TH, Coates ARM, Butcher PD, Hickman CJ, Shinnik TM. (1993) Mycobacterium tuberculosis expresses two chaperonin-60 homologs. Proc. Natl. Acad. Sci. U.S.A. 90: 26082612 . 